\title{
RELAÇÕES DE PRODUÇÃO NA PECUÁRIA LEITEIRA: UM ESTUDO DE CASO DAS RESPOSTAS DA PRODUÇÃO AOS PREÇOS MENSAIS ${ }^{1}$
}

\author{
Sílvio Ferreira Júnior ${ }^{2}$ \\ Erly Cardoso Teixeira ${ }^{3}$
}

Resumo - Pretende-se verificar as relações entre os insumos e produtos, no que se refere à substitutibilidade ou à complementariedade, bem como determinar os impactos de variações mensais nos preços dos produtos (leite e carne) e dos insumos (combustíveis, trabalho, concentrados) no sistema de produção no Brasil, que possui vacas de $2.000 \mathrm{~kg}$ a $3.000 \mathrm{~kg}$ de leite por lactação. Os resultados mostram que variações mensais nos preços pagos ou recebidos pelo produtor provocam mudanças mensais consideráveis nos mercados do leite e dos insumos considerados.

Palavras-chave: função translog de lucro, preços, pecuária leiteira.

\section{Introdução}

A pecuária leiteira no Brasil, até 1991, era apoiada pela regulamentação do Estado, principalmente por meio de controle dos preços e das importações. Tal prática, segundo Gomes (1999), trouxe conseqüências estruturais significativas e desfavoráveis à atividade, o que resultou na manutenção de baixos índices de produtividade, no baixo dinamismo empresarial e na pouca articulação dentro da cadeia. De acordo com Reis et al. (1993), a intervenção governamental não alcançou os objetivos de estímulo à produção e à produtividade, de estabilização da renda do

Recebido em 21/10/2004

Aceito em 28/03/2005

2 Economista, Mestre em Economia Aplicada e Doutorando em Economia Aplicada (Bolsista do CNPq). Universidade Federal de Viçosa - Departamento de Economia Rural, CEP 36570-000, Viçosa-MG. silviofj@hotmail.com

3 Professor titular do Departamento de Economia Rural da Universidade Federal de Viçosa. CEP 36570-000, Viçosa-MG. teixeira@ufv.br 
produtor e de melhoria na infra-estrutura produtiva; ao contrário, contribuiu para a pouca profissionalização do segmento produtivo.

A partir do início da década de 90, toda a cadeia do leite passou por mudanças significativas, em decorrência de três fatores principais (Gomes, 1999): a) Desregulamentação da atividade em 1991, que resultou na liberação do preço e na conseqüente queda deste, após quase meio século de tabelamento ${ }^{4}$; b) Maior abertura comercial, em especial, a implantação do Mercosul, que, por um lado, favoreceu as importações de lácteos e, por outro, ajudou a criar a cultura de competição; c) Estabilidade da economia brasileira com o Plano Real, que afetou, substancialmente, o agronegócio do leite. A combinação desses fatores aumentou a concorrência em todos os elos da cadeia do leite, especialmente para o produtor, que passou a competir com outros nos mercados regional, nacional e internacional.

Segundo Martins (2001), as transformações ocorridas ao longo da última década podem ser divididas em quatro tendências. A primeira delas é o aumento de $29,5 \%$ na produção nacional, de 1990 a 1996, contra 24,1\%, de 1980 a 1996. A segunda refere-se à mudança geográfica da produção nacional, visto que a região Sudeste, principal produtora de leite, vem perdendo espaço relativo na produção para a Centro-Oeste ${ }^{5}$. A terceira relaciona-se com a especialização do produtor, que se traduz em aumento de volume produzido e em redução de custos, por meio da mecanização, da adoção de novas tecnologias na alimentação e manejo, além da melhoria no padrão genético do rebanho ${ }^{6}$. A quarta e última tendência diz respeito à organização dos produtores; da típica condição reclamatória dos anos 80, as lideranças realizam reivindicações apoiadas em sólidos embasamentos técnicos, o que aumenta o poder de pressão e facilita o diálogo dos diferentes segmentos da cadeia com o governo ${ }^{7}$.

\footnotetext{
Segundo Homem de Melo (1999), houve queda significativa de $43 \%$ nos preços reais recebidos pelos produtores, entre 1989 e 1998

5 O Estado de Goiás quase duplicou sua produção, de 1990 a 1996. Atualmente, é o segundo maior produtor nacional, atrás de Minas Gerais, e o Estado de São Paulo caiu para a terceira posição no ranking (Martins, 2003; Zoccal e Fernandes, 2001).

6 Isto explica a relação, no longo prazo, de queda de preço e aumento da produção.

7 De acordo com Martins (2001), diante das facilidades de importação de leite e derivados até o ano de 1997, a Comissão de Agricultura e Política Rural da Câmara dos Deputados aceitou os argumentos da liderança dos produtores e propôs medidas que visem à inspeção sanitária e industrial de produtos importados.
} 
Atualmente, a atividade ocupa a $6^{\mathrm{a}}$ posição no ranking da agropecuária nacional, em termos de valor, e o $5^{\circ}$ no ranking mundial, em termos de quantidade (Martins, 2003). No entanto, o Brasil ainda está distante dos países que apresentam as maiores produtividades do rebanho e continua sendo um importador líquido de lácteos (Souza, 2000), o que indica que os ajustamentos necessários à melhoria da competitividade ainda estão em andamento.

Diante das transformações apontadas, a atividade leiteira nacional inserese, atualmente, num ambiente caracterizado por frequientes mudanças conjunturais, o que reflete em variações sucessivas nos preços dos insumos, uma vez que estes são dolarizados em grande parte, e na alta instabilidade nos preços pagos ao produtor, não só devido à sazonalidade como também à cartelização na captação do leite (Figueiredo Jr., 2002; Pagotto, 2002; Figueiredo Jr., 2003).

Dessa forma, o conhecimento das relações entre preços e quantidades de insumos e produtos no processo produtivo é de suma importância não só para o produtor, como também para os demais elos da cadeia (a montante e a jusante) e para o governo.

Pressupondo-se que a pecuária leiteira no Brasil seja gerida pelos princípios da eficiência econômica, seu objetivo é, portanto, a maximização de lucros. Isto ocorre quando, sob a lei dos rendimentos decrescentes, o valor do produto marginal de cada insumo utilizado na atividade é igual ao preço deste insumo e quando a receita marginal de cada produto é igual ao seu preço no mercado, a dado nível tecnológico e considerando que a atividade seja tomadora de preços de insumos e produtos.

Este trabalho objetivou caracterizar a estrutura de oferta de produtos e de demanda de insumos no sistema de produção no Brasil, que possui vacas de $2.000 \mathrm{~kg}$ a $3.000 \mathrm{~kg}$ de leite por lactação. Pretende-se verificar as relações entre insumos e produtos, no que se refere à substitutibilidade ou à complementariedade, bem como verificar os impactos das variações nos preços dos produtos (leite e carne) e dos insumos (combustíveis, trabalho, concentrados) neste sistema. 
Gurgel et al. (2000) realizaram estudo semelhante, ao analisarem as relações de produção na pecuária leiteira de Minas Gerais, considerando uma amostra de produtores que abrangia todos os níveis de produção (pequeno, médio e grande), no ano de 1995.

Entretanto, a atividade leiteira no Brasil caracteriza-se pela existência de diversos sistemas de produção alternativos, com diferentes combinações de insumos e diferentes níveis de produtividade. Assim, estudos que desconsideram este fato podem chegar a resultados poucos representativos para um sistema de produção específico, apresentando, portanto, pouca confiabilidade como parâmetro para tomada de decisão (Ferreira Júnior e Cunha, 2004).

Este trabalho, portanto, consiste num estudo de caso que permitirá fazer a comparação dos seus resultados com os de Gurgel et al. (2000). Outra contribuição relevante consiste na utilização de dados mensais, que permitirá obter informações a respeito das respostas imediatas da produção às variações mensais nos preços dos produtos e insumos.

\section{Metodologia}

A função de produção estabelece relação entre quantidades de insumos e quantidade máxima de produto, o que permite avaliar as respostas decorrentes de choques aplicados aos mercados de produtos, ou de fatores, sobre o sistema como um todo.

A teoria da dualidade mostra que é possível obter as ofertas de produtos e demandas de insumos como funções explícitas dos preços relativos, a partir de relações duais de lucro ou de custo, evitando-se pressuposições com respeito à função de produção. 
Seja a função de produção

$$
q=f(x) ; \quad q \text { e } x \in \mathrm{S}
$$

em que $q$ e $x$ são vetores de quantidades de produtos e de insumos variáveis, respectivamente, e fazem parte do conjunto de possibilidades $S$; a expressão $f$ é contínua, duplamente diferenciável, côncava e fechada em $q ; e x$ é estritamente crescente em $x$.

Sejam $p$ e $w$ vetores de preços de produtos e de insumos, respectivamente. Admitindo que os produtores sejam tomadores de preço nos mercados de insumos e produtos, a maximização do lucro (p) do produtor pode ser expressa por

Máx $\pi=p^{\prime} q-w^{\prime} x$, s.a $q=f(x)$.

As soluções de controle serão

$x^{*}=v(p, w)$,

$q^{*}=f(v(p, w))$,

em que $x^{*}$ é vetor de demandas de insumos e $q^{*}$, vetor de ofertas de produtos da maximização de lucro. A função objetivo otimizada - função indireta de lucro - será expressa por $\pi *(p, w)$.

A função indireta de lucro é contínua, duplamente diferenciável, convexa e fechada nos vetores de preços ( $p$ e $w$ ), estritamente crescente em $p$ e estritamente decrescente em $w$ e linear homogênea em $p$ e $w$. Observe que essas propriedades não dependem da tecnologia.

Neste trabalho utiliza-se a função indireta de lucro na forma funcional transcendental logarítmica (translog). A forma funcional translog permite diferentes graus de substitutibilidade e de retornos à escala, oferece uma 
aproximação de segunda ordem da série de Taylor para uma função de lucro qualquer, e ainda apresenta qualquer valor para as elasticidades de substituição entre quaisquer pares de insumos e produtos. Por ser expressa em logaritmos, a translog também facilita o cálculo das elasticidades-preço da demanda de fatores e oferta de produtos a partir das parcelas de lucro (Christensen et al., 1973; Hertel, 1984).

A função translog de lucro tem a seguinte forma matricial:

$\ln \pi=\alpha_{0}+\sum_{i} \alpha_{i} \ln \mathrm{P}_{\mathrm{i}}+1 / 2 \sum_{i} \sum_{j} \beta_{i j} \ln \mathrm{P}_{\mathrm{i}} \ln \mathrm{P}_{\mathrm{j}}$,

em que $\mathrm{P}_{\mathrm{i}}$ e $\mathrm{P}_{\mathrm{j}}$ correspondem aos preços dos produtos e dos insumos.

Diferenciando-se a função translog de lucro dos logaritmos dos preços dos produtos e dos fatores, por meio do Lema de Hotelling, obtêm-se as expressões das parcelas de lucro $\left(\mathrm{S}_{\mathrm{i}}\right)$ para cada produto ou insumos $\left(\mathrm{Y}_{\mathrm{i}}\right)$ :

$\frac{\partial \ln \pi}{\partial \ln P_{i}}=\frac{Y_{i} P_{i}}{\pi}=S_{i}=\alpha_{i}+\sum_{j} \beta_{\mathrm{ij}} \ln P_{j}$

em que $Y_{\mathrm{i}}$ é quantidade ótima do produto ou do insumo i. As parcelas de lucro são, portanto, as participações da receita de cada produto e da despesa de cada insumo no lucro total.

A expressão (6) é consistente com a hipótese de maximização de lucros, mas, para que satisfaça às propriedades de uma função de lucro, devemse impor restrições aos valores de seus parâmetros, com vistas em satisfazer às condições de simetria e de homogeneidade nos preços dos fatores e produtos. A função deve ainda satisfazer às condições de monotonicidade e convexidade, que não são impostas no modelo, mas são verificadas após a estimação. 
As condições de simetria e homogeneidade na função de lucro podem ser expressas por

$\beta_{\mathrm{ij}}=\beta_{\mathrm{ji}}, \quad \mathrm{i} \neq \mathrm{j}$

$\sum_{i} \alpha_{i=1}$;

$\sum_{j} \beta_{i j=} \sum_{i} \beta_{j i=0 .}$

A condição de monotonicidade implica parcelas estimadas positivas para produtos e negativas para fatores, enquanto a convexidade é examinada pelo Hessiano da matriz de parâmetros $\beta$, que deve ser positiva semidefinida, ou quando a matriz Hessiana, formada pelas elasticidades parciais de substituição, for positiva semidefinida (Hertel, 1984).

A função translog de lucro nem sempre satisfaz às condições de monotonicidade, globalmente. De acordo com Hertel (1984), é comum a obtenção de estimativas que violam a teoria econômica, devido a problemas relativos à agregação dos dados, à qualidade destes e, ainda, à própria vulnerabilidade da manutenção da hipótese de maximização de lucro. No entanto, se as condições forem satisfeitas localmente, ou seja, forem satisfeitas para uma gama de preços, os resultados serão consistentes com a maximização de lucros.

O sistema de parcelas de lucro pode ser estimado pelo Método de Zellner, para equações aparentemente não-relacionadas (SUR - Seemingly unrelated regressions), sujeito às restrições de homogeneidade e simetria (Greene, 1990).

A partir das equações de parcelas, podem ser obtidas as elasticidades parciais de substituição - ou seja, as elasticidades-preço diretas (Zii) e preço cruzadas da oferta de produtos (Zij) e as elasticidades-preço diretas $\left(\eta_{i i}\right)$ e preço cruzadas da demanda dos fatores $\left(\eta_{i j}\right)$ - pelas seguintes expressões derivadas em Binswanger (1974): 


$$
Z_{i i}=\frac{\beta_{i i}}{S_{i}}+S_{i}-1, Z_{i j}=\frac{\beta_{i j}}{S_{i}}+S_{j}, \eta_{i i}=\frac{\beta_{i i}}{S_{i}}+S_{i}-1, \eta_{i j}=\frac{\beta_{i j}}{S_{i}}+S_{j .}
$$

Os níveis de significância das elasticidades são avaliados por meio dos erros-padrão, obtidos a partir dos erros-padrão dos coeficientes estimados, conforme Reis et al. (1993):

$$
e p Z_{i i}=\frac{e p \beta_{i i}}{S_{i}} ; e p Z_{i j}=\frac{e p \beta_{i j}}{S_{i}} ; e p \eta_{i i}=\frac{e p \beta_{i i}}{S_{i}} ; e p \eta_{i j}=\frac{e p \beta_{i j}}{S_{i}} .
$$

A estimação do sistema de equações de parcelas de lucro requer que sejam selecionados os principais produtos e insumos envolvidos no sistema de produção. Neste estudo, o processo de seleção das variáveis procurou conciliar a importância de cada produto para a receita total e de cada insumo para o custo operacional total ${ }^{8}$, com disponibilidade de informações ao longo do período analisado. Assim, na função translog de lucro, na forma expressa em (5), incluirão os preços dos produtos leite (L) e carne $(\mathrm{C})^{9}$, os preços dos insumos combustiveis $(\mathrm{K})$, trabalho $(\mathrm{M}) \mathrm{e}$ concentrados (A), e o lucro total da atividade, considerado como a diferença entre o valor das receitas provenientes das vendas de leite e carne e o valor das despesas provenientes dos gastos com combustíveis, trabalho e concentrados.

O sistema de equações parciais constitui uma matriz singular e, portanto, não-inversível. Esse problema é resolvido ao aplicar, novamente, a restrição de homogeneidade, para eliminar uma das equações, que terá seus parâmetros obtidos por diferença, observando-se as condições de homogeneidade e simetria impostas ao modelo (Berndt, 1997).

Neste estudo, eliminou-se, primeiramente, a equação de parcela de concentrados, normalizando as equações de parcela do leite, da carne, dos combustíveis e do trabalho pelo preço do insumo concentrados,

\footnotetext{
Na média do período analisado, os produtos considerados correspondem a 96\% da receita total, enquanto os insumos correspondem a $68 \%$ do custo operacional total.

9 De acordo com Reis et al. (1993) e Gurgel et al. (2000), a pecuária leiteira é uma atividade de produção conjunta que utiliza os mesmos insumos para a produção de leite e carne. A carne deve ser considerada como o agregado das vendas de animais para o abate e para as atividades de cria e recria.
} 
obtendo-se as seguintes equações:

$$
\begin{aligned}
& S_{L}=\alpha_{L}+\beta_{L L} \ln \left(P_{L} / P_{A}\right)+\beta_{L C} \ln \left(P_{C} / P_{A}\right)+\beta_{L K} \ln \left(P_{K} / P_{A}\right)+\beta_{L M} \ln \left(P_{M} / P_{A}\right), \\
& S_{C}=\alpha_{C}+\beta_{C L} \ln \left(P_{L} / P_{A}\right)+\beta_{C C} \ln \left(P_{C} / P_{A}\right)+\beta_{C K} \ln \left(P_{K} / P_{A}\right)+\beta_{C M} \ln \left(P_{M} / P_{A}\right), \\
& S_{K}=\alpha_{K}+\beta_{K L} \ln \left(P_{L} / P_{A}\right)+\beta_{K C} \ln \left(P_{C} / P_{A}\right)+\beta_{K K} \ln \left(P_{K} / P_{A}\right)+\beta_{K M} \ln \left(P_{M} / P_{A}\right), \\
& S_{M}=\alpha_{M}+\beta_{M L} \ln \left(P_{L} / P_{A}\right)+\beta_{M C} \ln \left(P_{C} / P_{A}\right)+\beta_{M K} \ln \left(P_{K} / P_{A}\right)+\beta_{M M} \ln \left(P_{M} / P_{A}\right)
\end{aligned}
$$

em que $S_{i}$ é parcela de lucro do produto, - $S_{i}$, parcela de lucro do insumo; e $\mathrm{P}_{\mathrm{i}}$, preços do produto ou fator, sendo $\mathrm{i}=$ leite $(\mathrm{L})$, carne $(\mathrm{C})$, combustíveis $(\mathrm{K})$, trabalho $(\mathrm{M})$ e concentrados $(\mathrm{A})$.

Ao fazer a normalização, os parâmetros da equação da parcela de concentrados podem ser estimados residualmente; nesse caso, não se teria o nível de significância estatística deles. Para contornar esse problema, montou-se um segundo sistema de equações normalizado pelo preço do leite e, em seguida, elaborou-se um terceiro sistema normalizado pelo preço dos combustíveis. Esse procedimento, também realizado por Castro e Teixeira (2003), permite obter o nível de significância de todos os parâmetros do sistema normalizado pelo fator trabalho.

Deve-se observar que, após a normalização, os parâmetros $\beta_{\mathrm{ij}}$ estimados incorporaram a relação de preços $\mathrm{P}_{\mathrm{i}} / \mathrm{P}_{\mathrm{A}}$. Portanto, as elasticidades parciais de substituição, obtidas desses parâmetros, são interpretadas como a variação na quantidade ofertada de produto ou demandada de insumo a partir da variação na relação de preços $\mathrm{P}_{\mathrm{i}} / \mathrm{P}_{\mathrm{A}}$.

Os dados mensais utilizados neste estudo abrangem o período de janeiro de 2001 a dezembro de 2002 e foram obtidos das planilhas de custo divulgadas no Anuário da Pecuária Brasileira, da FNP Consultoria e AgroInformativos, com exceção do preço da carne, que foi fornecido pelo Instituto de Pesquisa Econômica Aplicada (IPEA). As planilhas selecionadas correspondem ao sistema de produção constituído de vacas de $2.000 \mathrm{~kg}$ a $3.000 \mathrm{~kg}$ de leite por lactação. 
A parcela de lucro com leite (carne) é dada pela razão entre a receita da venda de leite (venda de animais) e o lucro total da atividade leiteira. A parcela de lucro com trabalho é dada pela relação entre o gasto com mão-de-obra contratada (para manejo do rebanho e para manutenção de pastagens e de benfeitorias) e o lucro total. A parcela de lucro com concentrados é a relação entre o fluxo dos gastos com a compra de rações e o lucro total obtido na atividade. A relação entre o fluxo de gastos com combustíveis e o lucro total refere-se à parcela de lucro com combustíveis derivados do petróleo.

Os preços médios do leite, do trabalho, de concentrados e de combustíveis referem-se, para cada item, à razão entre o valor total e a quantidade. Para o preço da carne utilizou-se, como proxy, o preço mensal pago ao produtor do boi magro. Utilizou-se, como deflator, o IGP-DI (agosto de $1994=100)$.

\section{Resultados e discussões}

Os resultados das estimativas dos parâmetros das equações de parcelas de lucro estão apresentados na Tabela 1. As equações satisfazem às restrições de simetria e homogeneidade, como pode ser verificado pelos coeficientes estimados $\left(\beta_{\mathrm{ij}}=\beta_{\mathrm{ji}}\right)$ e pela soma das linhas iguais a zero $\left(\sum_{j} \beta_{i j=} \sum_{i} \beta_{j i=0 ;} \sum_{i} \alpha_{i=1}\right)$. 
Tabela 1. Estimativas dos coeficientes do sistema de parcelas de lucro para o sistema de produção de leite, janeiro de 2001 a dezembro de 2002

\section{2.}

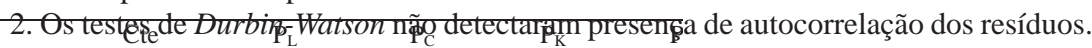

${ }^{* *}$ Significativo a $1 \% ;{ }^{*}$ significativo $5 \%$; ${ }^{\text {ns }}$ não-significativo.

\begin{tabular}{cc|c|ccc}
\multicolumn{6}{c}{ Fonte: Dados da pesquisa. } \\
\hline $\mathrm{S}_{\mathrm{L}}$ & $15,6938^{* * *}$ & $3,0692^{* *}$ & $-0,6592^{* *}$ & $-0,2579^{* *}$ & $-1,2$ \\
& $(1,7436)$ & $(0,3886)$ & $(0,2369)$ & $(0,0855)$ & $(0,2$
\end{tabular}

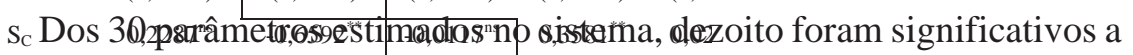

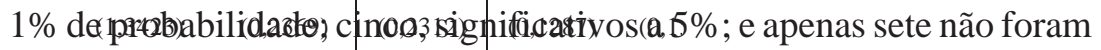

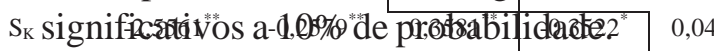
$(0,6874)$
$(0,0855)$
$(0,1287)$
$(0,1785)$
$(0,0$ :

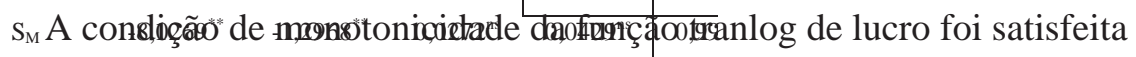
globalmente, uma6rez qwe trodas (as503rcellas estimadas foram positivas

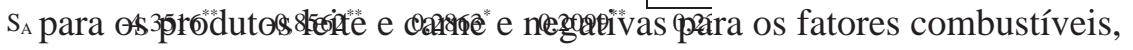

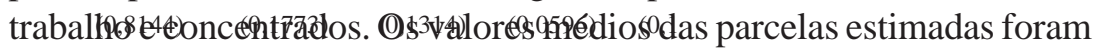

Somde 2,2690) 0,5798, para leite e carne, e de -0,1588, -1,0684 e -0,6215, para combustíveis, trabalho e concentrados, respectivamente. 
Os coeficientes apresentados na Tabela 1 são utilizados no cálculo das elasticidades parciais de substituição, apresentadas na Tabela 2, que permitem descrever a estrutura de oferta dos produtos e da demanda de fatores para o sistema de produção.

Percebe-se, novamente, que a restrição de homogeneidade foi satisfeita, ao se observar que o somatório das elasticidades em cada equação é igual a zero. A condição de convexidade foi satisfeita para o produto leite, pelo valor positivo da elasticidade-preço direta da oferta de leite, e para os fatores trabalho e concentrados, pelos valores negativos da elasticidade-preço direta de suas respectivas demandas. Entretanto, esta condição não foi satisfeita globalmente, uma vez que a elasticidade-preço direta da oferta de carne foi negativa. A elasticidade-preço direta da demanda de combustíveis foi positiva, porém seu valor não foi estatisticamente diferente de zero. Pode-se dizer, entretanto, que a condição de convexidade da função translog de lucro foi satisfeita localmente.

A Tabela 2 permite evidenciar que aumentos de $1 \%$ no preço do leite induzem ao aumento da quantidade produzida de leite mais que proporcionalmente ao aumento no preço. A elasticidade-preço direta de $2,62 \%$ caracteriza o sistema produtivo como de oferta expressivamente elástica. 
Tabela 2. Estimativas das elasticidades-preço diretas e elasticidadespreço cruzadas da oferta e da demanda do sistema de produção de leite, janeiro de 2001 a dezembro de 2002

\begin{tabular}{cccccr}
\hline Quant. & & \multicolumn{3}{c}{ Preço } \\
& Leite & Carne & Combustíveis & Trabalho & Conce \\
\hline \multirow{3}{*}{ (L) } & $(\mathrm{L})$ & $(\mathrm{C})$ & $(\mathrm{K})$ & $(\mathrm{M})$ & ( \\
& $2,6217^{* *}$ & $0,2893^{* *}$ & $-0,2725^{* *}$ & $-1,6399^{* *}$ & $-0,9$ \\
& $(0,1713)$ & $(0,1044)$ & $(0,0377)$ & $(0,1152)$ & $(0,0$ \\
(C) & $1,1321^{* * *}$ & $-0,4403^{* *}$ & $0,4588^{*}$ & $-1,0215^{* *}$ & $-0,1$ \\
& $(0,4086)$ & $(0,0202)$ & $(0,2220)$ & $(0,2725)$ & $(0,2$ \\
(K) & $3,8927^{* *}$ & $-1,6764^{* *}$ & $1,0586^{\mathrm{ns}}$ & $-1,3385^{* *}$ & $-1,9$ \\
& $(0,5383)$ & $(0,8103)$ & $(0,1238)$ & $(0,3167)$ & $(0,3$ \\
(M) & $3,4828^{* * *}$ & $0,5544^{* *}$ & $-0,1990^{* *}$ & $-2,9970^{* * *}$ & $-0,8$ \\
& $(0,2446)$ & $(0,1479)$ & $(0,0471)$ & $(0,2361)$ & $(0,1$ \\
(A) & $3,6467^{* * *}$ & $0,1191^{\mathrm{ns}}$ & $-0,4966^{* *}$ & $-1,4464^{* *}$ & $-1,8$ \\
& $(0,2853)$ & $(0,2114)$ & $(0,0959)$ & $(0,1838)$ & $(0,1$ \\
\hline
\end{tabular}

1. Erros-padrão entre parênteses.

${ }^{* *}$ Significativo a $1 \%$; ${ }^{*}$ significativo $5 \%$; ${ }^{\text {ns }}$ não-significativo.

Fonte: Dados da pesquisa.

Vale lembrar que os dados utilizados neste estudo são mensais. Portanto, pode-se dizer que a produção, no sistema considerado, responde rapidamente às variações de curto prazo no preço do leite. Conforme Gurgel et al. (2000), sistemas de produção mais tecnificados, com maior utilização de insumos modernos, são mais elásticos, quanto à oferta. A despeito de haver, nas diversas regiões brasileiras, grande heterogeneidade de produtores e técnicas produtivas, com predominância numérica de produtores tradicionais (Gomes, 1999), percebe-se, a partir do alto valor da elasticidade-preço direta da oferta de leite, que o sistema em análise se caracteriza pela utilização de técnicas modernas de produção.

A elasticidade-preço cruzada da oferta de leite, em relação ao preço da carne, indica relação de complementaridade entre eles, uma vez que o aumento do preço da carne (medido pelo preço dos animais) leva o produtor a intensificar a produção de leite.

A elasticidade-preço direta da oferta de carne apresentou sinal contrário 
ao esperado pela teoria, enquanto sua elasticidade-preço cruzada da oferta, em relação ao preço do leite, confirmou a relação de complementaridade entre os produtos. $\mathrm{O}$ alto valor desta última elasticidade (1,13), em relação à elasticidade-preço cruzada da oferta de leite em relação ao preço da carne $(0,29)$, evidencia maior sensibilidade à variação no preço do leite do que no preço de venda do animal. De fato, o leite é o produto principal da atividade, sendo a carne consequiência do primeiro e uma forma de diversificação da renda do produtor (Reis et al., 1993; Gurgel et al., 2000).

Com relação aos resultados das elasticidades-preço cruzadas da oferta do leite em relação ao preço dos insumos, percebe-se que a atividade leiteira é sensível às variações nos preços destes, visto que se reduz a produção de leite quando ocorre aumento nos preços dos fatores combustíveis, trabalho e concentrados. Destaca-se o valor relativamente alto da elasticidade-preço cruzada da oferta em relação ao preço do trabalho $(-1,64)$, ao evidenciar que a produção leiteira foi relativamente mais sensível às variações mensais no preço deste fator, em comparação às variações nos preços dos combustíveis $(-0,27)$ e dos concentrados $(-$ $1,00)$.

Quanto às elasticidades-preço cruzadas da oferta de carne em relação ao preço dos insumos, percebe-se que a oferta deste produto diminuiu na mesma proporção do aumento no preço do trabalho (elasticidade próxima da unitária), mas aumentou com o aumento no preço dos combustíveis utilizados na atividade $(0,46)$, constituindo, de acordo com Hertel (1984), uma relação de regressividade entre o produto e o fator. A elasticidadepreço cruzada da oferta de carne, em relação ao preço dos concentrados, foi estatisticamente não-significativa a $10 \%$.

Os resultados mostram que tanto a produção de leite quanto a de carne foram mais sensíveis às variações no preço do fator trabalho do que às variações no preço do insumo concentrados e mais sensíveis às variações no preço dos concentrados do que às variações no preço do insumo combustíveis. De fato, o trabalho é o fator agente que realiza e, ou, 
operacionaliza a atividade, de modo que a proporção relativa entre as elasticidades encontradas evidencia uma característica importante da atividade leiteira.

No que se refere à demanda dos fatores de produção, as elasticidadespreço diretas da demanda de trabalho e de concentrados foram de $3,00 \%$ e $-1,82$, respectivamente, enquanto a demanda de combustíveis não apresentou elasticidade-preço estatisticamente significativa.

Os valores expressivos dessas elasticidades são explicados pelo peso significativo desses insumos no custo operacional total da atividade. Conforme salientou Camargo (2002), na quase totalidade das propriedades leiteiras do Brasil, os fatores alimentação e trabalho compõem-se, respectivamente, de 50 a $60 \%{ }^{10}$ e de 10 a $20 \%$ do custo total da atividade. Esse alto grau de resposta da produção às variações nos preços dos insumos permite caracterizar o sistema em análise como moderno, uma vez que a utilização de tecnologias modernas está, em geral, relacionada com maior flexibilidade do sistema produtivo, conforme salientaram Fulginiti e Perrin (1990).

Com relação aos sinais das elasticidades-preço cruzadas das demandas dos insumos, percebe-se que todos apresentaram sinais negativos, o que indica uma característica de complementaridade entre os insumos utilizados na atividade. Pode-se dizer que esses insumos são utilizados em proporções fixas; dessa forma, o aumento no preço de qualquer um deles provocará redução na quantidade demandada de todos os três fatores, conjuntamente.

A relação de complementaridade entre os fatores pode ainda ser verificada por meio dos valores das elasticidades-preço cruzada das demandas dos três fatores em relação ao preço do leite, que são bem próximos e bastante expressivos $(-3,89,-3,48$ e -3,65 para combustíveis, trabalho e concentrados, respectivamente), ou seja, um aumento de $1 \%$

${ }^{10}$ Dessas percentagens, cerca de 40 a $50 \%$ refere-se ao uso de concentrados (rações) e 10 a $20 \%$ correspondem aos alimentos volumosos. 
no preço do leite provocará aumento de cerca de $3 \%$ nas quantidades demandadas de combustíveis, de trabalho e de concentrados.

Quanto às elasticidades-preço cruzadas das demandas dos fatores em relação ao preço da carne, observa-se que a referente à demanda de trabalho apresentou sinal positivo, enquanto na demanda de concentrados a elasticidade foi estatisticamente não-significativa, mas com sinal positivo, e na demanda de combustíveis ela foi alta e negativa, o que indica regressividade entre a demanda do fator combustíveis e a oferta de carne.

Pode-se observar, pelos resultados das Tabelas 1 e 2, que os parâmetros estimados referentes ao produto carne foram, em geral, menos expressivos e menos consistentes do que os estimados para leite. Este fato é mais uma evidência de que o sistema produtivo analisado é especializado na produção de leite. Assim, o gerenciamento racional da atividade, na ótica da maximização de lucros, estaria mais voltado para o produto leite, enquanto o abate ou a venda de animais seria um complemento da atividade.

Quanto aos demais parâmetros, os resultados deste estudo foram semelhantes aos observados no estudo de Gurgel et al. (2000), no que se refere aos sinais e à proporção relativa entre as diversas elasticidades. Entretanto, no que concerne aos valores desses parâmetros, as elasticidades atuais foram, no geral, significativamente maiores que as encontradas naquele estudo. A título de comparação, as elasticidadespreço diretas daquele estudo foram de 1,33, -1,60 e -1,32, para leite, trabalho e concentrados, respectivamente, contra 2,67,-3,00 e -1,82, deste estudo. As elasticidades-preço da demanda dos fatores, em relação ao preço do leite, foram, respectivamente, de 2,16 e 2,37, para trabalho e concentrados, contra 3,48 e 3,65 , deste estudo.

Portanto, percebe-se que, devido à grande heterogeneidade da atividade leiteira no Brasil, recomenda-se que o cálculo de elasticidades neste setor seja feito nos sistemas de produção, para que os resultados sejam mais confiáveis e úteis à tomada de decisão dos diversos agentes envolvidos na cadeia do leite. 


\section{Conclusões}

Este trabalho procurou caracterizar a estrutura de oferta de produtos e de demanda de insumos no sistema de produção leiteira no Brasil, que possui vacas de $2.000 \mathrm{~kg}$ a $3.000 \mathrm{~kg}$ de leite por lactação, por meio da estimação de um sistema de equações parciais de lucro dos produtos leite e carne e dos insumos combustíveis, trabalho e concentrados.

Os produtos leite e carne são complementares no sistema considerado, dadas as elasticidades-preço cruzadas positivas, verificadas em ambos. A oferta do leite é elástica em relação ao preço; inelástica em relação ao preço dos combustíveis; elástica em relação ao preço do trabalho; e elasticidade unitária em relação ao preço de concentrados. A oferta da carne é inelástica-negativa em relação ao preço; inelástica-positiva em relação ao preço dos combustíveis (regressividade); elástica em relação ao preço do trabalho; e elasticidade não-significativa em relação ao preço de concentrados.

Os sinais negativos das elasticidades-preço cruzadas das demandas entre os preços dos insumos, bem como os valores positivos e aproximados dessas elasticidades em relação ao preço do leite, evidenciam o caráter de complementaridade entre os insumos combustíveis, trabalho e concentrados utilizados na atividade, o que indica que estes devem ser utilizados em proporções fixas, para que se tenha efeito na quantidade de leite produzida.

Resultados mais frágeis para o produto carne indicam que o abate ou a venda de animais é um complemento residual da atividade, e não está fortemente baseado nos princípios da maximização de lucros. No entanto, os resultados para o produto leite mostram que o gerenciamento racional da atividade está voltado para a produção de leite. Esses resultados permitem evidenciar o alto grau de especialização e modernização do sistema de produção analisado, que, ao ser gradativamente adotado pelos diversos produtores no Brasil, permitirá que o país tenha aumentos expressivos na produção e na produtividade. 
A obtenção das elasticidades, por meio da utilização de dados mensais, permite que se tenham informações sobre as respostas imediatas da produção às variações mensais nos preços dos produtos e insumos. Essas informações são importantes para o produtor e para os demais agentes envolvidos na cadeia, uma vez que variações mensais nos preços pagos ou recebidos pelo produtor provocam mudanças não só imediatas, mas também expressivas, nos mercados do leite e de insumos considerados.

\section{Referências}

BINSWANGER, H.P. A cost function approach to the measurement of elasticities of factor demand and elasticities of substitution. The American Journal of Agricultural Economics. V. 56, n², p. 377-86, 1974.

CAMARGO, A.C. Condições para produzir leite a baixo custo. In: Anuário da Pecuária Brasileira - ANUALPEC. São Paulo: FNP Consultoria e Agroinformativo, 9 ed., p.203-204, 2002.

CASTRO, E.R.; TEIXEIRA, E.C. Efeito da desvalorização cambial na oferta, no preços dos insumos e na relação entre os fatores na cultura do café. In: CONGRESSO BRASILEIRO DE ECONOMIA E SOCIOLOGIA RURAL, 41, 2003, Juiz de Fora. Anais... Juiz de Fora: SOBER, 2003. (CD-ROM).

CHRISTESEN, L.R.; JORGENSON, D.W.; LAW, L.J. Transcendental logarithmic production function frontiers. Review of Economics and Statistics, Cambridge, v.55, n.1, p.25-45, fev. 1973.

BERNDT, E. R. The practice of econometrics classic and contemporary. Addison-Wesley, 1991.

FERREIRA JÚNIOR, S.; CUNHA, N.R.S. Eficiência técnica na atividade leiteira de Minas Gerais: um estudo a partir de três sistemas de produção. Organizações Rurais e Agroindustriais. Lavras: UFLA, v.6, n.2, p. 46-60, Jul/Dez 2004. 
FIGUEIREDO JÚNIOR, G.A. Diferentes estratégias para enfrentar a crise na agropecuária leiteira. In: Anuário da Pecuária Brasileira ANUALPEC. São Paulo: FNP Consultoria e Agroinformativo, 9 ed., p.208-210, 2002.

FIGUEIREDO JÚNIOR, G.A. Produtores de leite com perspectivas animadoras. In: Anuário da Pecuária Brasileira - ANUALPEC. São Paulo: FNP Consultoria e Agroinformativo, 10 ed., p.215-216, 2003.

FULGINITI, L.E.; PERRI, R.K. Argentine agricultural policy in a multipleimput, multiple-output framework. American Journal of Agricultural Economics, Ames, v.72. n.2, p.279-288, may, 1990.

GOMES, A. P. Impactos das transformações da produção deleite no número de produtores e requerimentos da mão-de-obra e capital. Viçosa, MG: UFV, 1999. 161 p. Tese (Doutorado em Economia Rural) - Universidade Federal de Viçosa, 1999.

GREENE, W.H. Econometrics analysis. 2.ed., New York: McMillan, 1990, 783p.

GURGEL, A.C.; SANTOS, F.A.A.; TEIXEIRA, E.C..Relações de produção na pecuária leiteira de Minas Gerais - 1995. Revista de Economia e Sociologia Rural, v.38, n.4, p.29-51, out/dez, 2000.

HERTEL, T.W. Applications of duality and flexible functional forms: the case of the multiproduct firm. West Lafayette, Agricultural Experimentation Station, 1984. 46p. (Research Bulletin, 980).

HOMEM DE MELO, F. Leite: a difícil formulação de uma política comercial. Revista de Economia e Sociologia Rural, Brasília, v.37, n.4, p.33-44, 1999.

MARTINS, M.C. Agronegócio do leite. Disponível em: www.cnpgl.embrapa.br/jornal/informe 2003. Acessado em: 01 jun. 2003. 
MARTINS, P.C. Efeitos de políticas públicas sobre a cadeia produtiva do leite em pó. In: VIEIRA, R.C.M.T., et al. (Eds). Cadeias produtivas no Brasil: análise da competitividade. Brasília: EMBRAPA, 2001. 469 p.

PAGOTTO, D.S. Estratégia de produção de volumosos. In: Anuário da Pecuária Brasileira - ANUALPEC. São Paulo: FNP Consultoria e Agroinformativo, 9 ed., p.211-212, 2002.

REIS, R.P; TEIXEIRA, E.C; LIMA, J.E. O mercado de leite: política de intervenção e estrutura produtiva. Revista de Economia e Sociologia Rural.v.31, n.3, p.215-229, jul./set. 1993.

SOUZA, D.P.H. Análise da estrutura de custo e preço de sobrevivência dos principais sistemas de produção de leite. Viçosa, MG: UFV, 2000. 85p. Dissertação (Mestrado em Economia Rural), Universidade federal de Viçosa, 2000.

ZOCCAL, R., FERNANDES, E. N. Mudanças na pecuária de leite. Informe Econômico do Leite. EMBRAPA, Brasília, ano 1, n.1, dez. 2001.

Abstract - It is intended to verify the relations between input and products concerning to replaceability or complementarity, as well as the impacts of monthly variations on product prices (milk and meat) and input (fuels, work, concentrate) in the production system in Brazil that possess cows of 2.000 to 3.000 kilograms of milk for nursing. The results show that monthly variations in prices paid or received by producers cause considerable monthly changes in the markets for milk and the input considered.

Keywords: profit translog function, prices, dairy cattle. 